
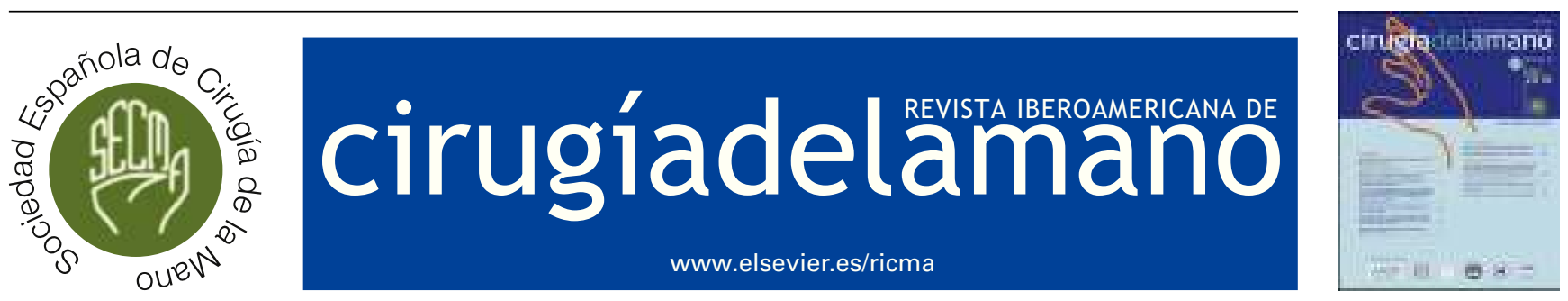

ARTÍCULO DE ACTUALIZACIÓN

\title{
Artrosis de la articulación radiocubital distal
}

\author{
F. Polo Simón ${ }^{a, *}$, E.P. Zancolli ${ }^{a}$, L. Vicentela Iturrieta ${ }^{a}$, P.S. Rotella ${ }^{b}$ y L.R. Scheker ${ }^{a}$
}

\author{
a Christine M. Kleinert Institute for Hand and Microsurgery, Louisville, KY, Estados Unidos \\ b Sanatorio del Norte - San Miguel de Tucumán, Tucumán, Argentina
}

Recibido el 12 de noviembre de 2015; aceptado el 13 de enero de 2016

Disponible en Internet el 9 de marzo de 2016

\section{PALABRAS CLAVE \\ Articulación radiocubital distal; Artrosis radiocubital distal; \\ Fosa sigmoidea; Artrosis; \\ Prótesis radiocubital distal}

\section{KEYWORDS}

Distal radio-ulnar joint; Distal radio-ulnar joint arthritis; Sigmoid notch; Arthritis; Distal radio-ulnar joint prosthesis
Resumen La articulación radiocubital distal (ARCD) es una de las articulaciones que más ha sido estudiada en los últimos años debido a su relevancia en la funcionalidad de la muñeca y del miembro superior en su conjunto. El diagnóstico y tratamiento de su patología supone un interesante reto para el cirujano de mano, especialmente la degeneración artrósica.

El tratamiento de la artrosis de la ARCD puede ser conservador o quirúrgico. El objetivo del tratamiento conservador es la disminución del dolor y la optimización de la función articular hasta que la cirugía se hace inevitable. Con respecto al tratamiento quirúrgico, se han descrito multitud de procedimientos diferentes que reflejan la variedad del espectro patológico de la ARCD. El mejor conocimiento de la anatomía y la biomecánica de la ARCD y los avances en la tecnología han convertido al reemplazo protésico en la tendencia actual para el tratamiento de la artrosis de la ARCD.

En este artículo, tratamos el diagnóstico y las opciones terapéuticas relativas a la artrosis de esta articulación de la fosa sigmoidea del radio y el cúbito distal basados en nuestra experiencia y en la revisión de la literatura actual.

(c) 2016 SECMA. Publicado por Elsevier España, S.L.U. Este es un artículo Open Access bajo la licencia CC BY-NC-ND (http://creativecommons.org/licenses/by-nc-nd/4.0/).

Osteoarthritis of the distal radio-ulnar joint

Abstract The distal radio-ulnar joint (DRUJ) is one of the most studied joints over the last few years due to its important role in wrist and upper limb function. Evaluation and management of DRUJ problems present a challenging issue for Hand Surgeons, specially DRUJ arthritis.

Management of DRUJ arthritis can be non-surgical or surgical. The goal of the non-surgical management is to address pain and optimise joint function before surgery becomes unavoidable. As far as surgical management is concerned, many procedures have been described reflecting the varying spectrum of pathology that is encountered. Prosthetic replacement of the

\footnotetext{
* Autor para correspondencia.

Correo electrónico: drfpolo@hotmail.com (F. Polo Simón).
} 
distal radioulnar joint is a recent trend for the management of arthritic distal radioulnar joints because of a better understanding of the distal radio-ulnar joint anatomy and biomechanics and technological advances.

In this article we discuss the diagnostic and management options for the arthritis at the articulation of the sigmoid notch and the distal ulna based on our broad experience and the current literature review.

(c) 2016 SECMA. Published by Elsevier España, S.L.U. This is an open access article under the CC BY-NC-ND license (http://creativecommons.org/licenses/by-nc-nd/4.0/).

\section{Introducción}

La articulación radiocubital distal (ARCD) es una de las articulaciones que más ha sido estudiada en los últimos años debido a su relevancia en la funcionalidad de la muñeca y del miembro superior en conjunto. Su compleja estructura y biomecánica entrañan una notable dificultad en el diagnóstico y tratamiento de su patología y hacen que estos supongan un interesante reto para el cirujano de la mano.

La patología de la ARCD se puede dividir fundamentalmente en 3 grandes grupos: impactación cubitocarpiana, inestabilidad e incongruencia y artrosis.

El propósito del presente artículo es hacer una actualización sobre el diagnóstico y las opciones terapéuticas de la artrosis de la ARCD.

La artrosis de la ARCD puede deberse, al igual que en otras áreas anatómicas, tanto a causas primarias como a causas secundarias. La artrosis primaria es más común en mujeres y se asocia con una varianza cubital positiva ${ }^{1}$. Las causas secundarias son muy numerosas, pero tienen como denominador común la incongruencia y/o la inestabilidad de la articulación como resultado de agresiones directas o indirectas (fracturas de extremidad distal del radio, lesiones de los ligamentos radiocubitales distales, infecciones, artritis reumatoide, etc.).

\section{Diagnóstico de la artrosis de la articulación radiocubital distal}

Como la mayoría de las patologías del miembro superior, el diagnóstico se basa fundamentalmente en el examen físico, complementándolo con el uso de pruebas de imagen, principalmente las radiografías.

\section{Examen físico}

El examen físico comienza con la inspección de la muñeca sintomática del paciente una vez terminada la anamnesis. En la inspección puede observarse, por ejemplo, cicatrices de cirugías previas, eritema, edema o la prominencia dorsal de la cabeza del cúbito. Es importante, al evaluar la articulación radiocubital distal, realizarlo comparativamente. Luego se continúa con la palpación, identificando ciertos puntos de referencia anatómicas tales como la cabeza del cúbito y su estiloides, el hueso piramidal, pisiforme, el ligamento lunopiramidal, el tendón flexor carpi ulnaris (FCU) y el extensor carpi ulnaris (ECU), y las inserciones del complejo fibrocartílago triangular (CFCT). El examinador debe buscar el punto doloroso mediante la palpación suave y compararlo con el lado opuesto. Localizado el punto anatómico del dolor, el examinador puede comenzar a realizar pruebas en busca de signos específicos de distintas patologías que afectan la ARCD. Es importante recordar que la compresión del nervio cubital a nivel del codo también puede presentarse como un dolor referido en la muñeca en su lado cubital. Este cuadro debe sospecharse al abordar el dolor en el lado cubital de la muñeca, para realizar el correcto diagnóstico diferencial.

Existen 2 maniobras relevantes en el examen físico enfocadas al diagnóstico de artrosis de la ARCD. En la primera se le pide al paciente que coloque su codo sobre la mesa con el antebrazo perpendicular a la mesa. El examinador aplica una fuerza de cizallamiento entre el radio y el cúbito con el antebrazo en pronación completa y luego en supinación completa. El grado de desplazamiento entre el radio y el cúbito en pronación y supinación máxima demuestra la presencia o no de integridad de los ligamentos dorsales y palmares radiocubital distal, respectivamente. La comparación con el lado contralateral es crucial. Durante esta prueba, la fuerza de cizallamiento y compresión entre el radio y el cúbito puede generar dolor, sugiriendo signos de incongruencia y artrosis radiocubital distal.

En la segunda maniobra se elimina el efecto de la gravedad en el lado cubital de la mano. Se le pide al paciente que coloque ambos codos pegados al cuerpo flexionados a $90^{\circ}$. El examinador aplica presión hacia abajo sobre las muñecas mientras se le pide al paciente alternativamente movimientos de pronosupinación de los antebrazos resistiendo la fuerza ejercida por el médico. Esta maniobra carga la ARCD durante su movimiento rotacional, permitiendo la translación entre el radio y el cúbito, mientras que se elimina la gravedad sobre el lado cubital de la muñeca. En presencia de incongruencia o artrosis de la ARCD, el paciente siente dolor durante la rotación del radio sobre la cabeza del cúbito o cede a la presión por parte del examinador. En esta prueba, la fuerza aplicada a la articulación pone en evidencia el desgaste articular y roce existente entre la cabeza del cúbito y la cavidad sigmoidea.

\section{Radiografías}

Durante el examen radiográfico de la ARCD consideramos importante evaluar: 1) la varianza cubital, 2) la configuración articular, 3) el pinzamiento articular bajo carga y 4) el desgaste articular. Las radiografías posteroanteriores (PA) de 


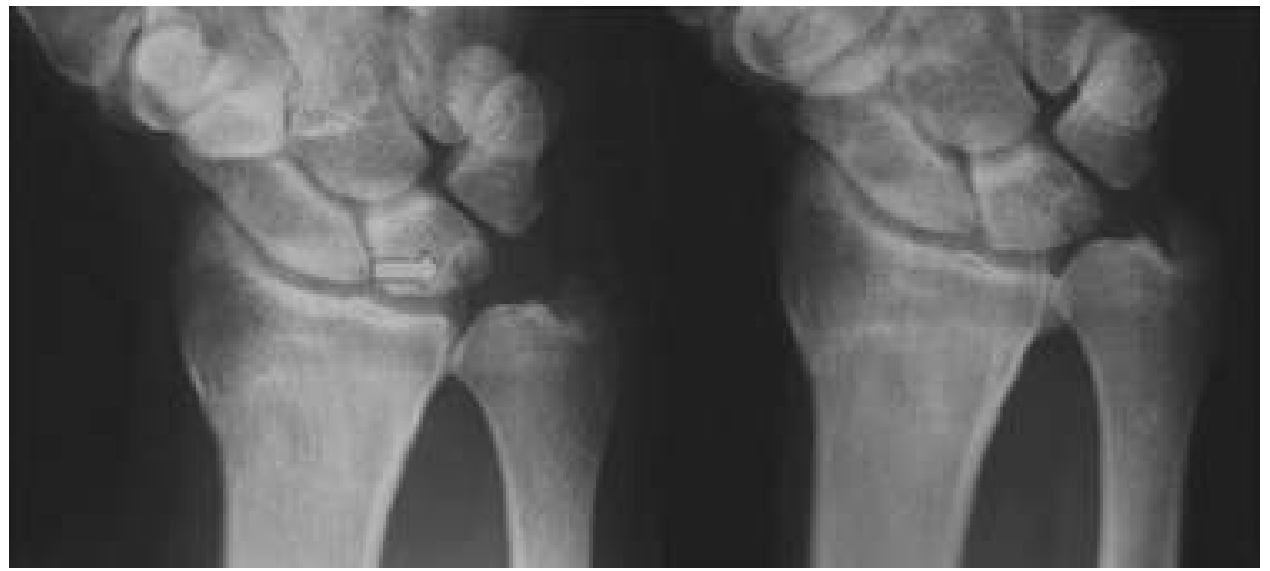

Figura 1 Radiografía anteroposterior de muñeca: la flecha indica una imagen radiolúcida en el borde proximal y cubital del semilunar característico del pinzamiento cubitocarpiano (izquierda). Evidente varianza cubital positiva (cúbito plus) con la muñeca en pronación con el puño cerrado en el mismo paciente (derecha). Cortesía del Christine M. Kleinert Institute for Hand \& Microsurgery.

muñeca deben realizarse sobre una superficie plana con el hombro en abducción de $90^{\circ}$ y el codo en flexión de $90^{\circ}$. La radiografía lateral se realiza con el hombro en aducción, el codo en $90^{\circ}$ de flexión y el antebrazo en posición neutra. En la radiografía lateral el pisiforme debe alinearse con el tercio distal del escafoides.

La evaluación de la varianza cubital es parte fundamental del examen radiográfico. Esta puede presentarse como neutra (radio y cúbito de igual longitud), positiva o cúbito plus (extremo distal del cúbito distal al del radio) y negativa o cúbito minus (extremo distal del cúbito proximal al del radio). Durante el examen radiográfico es importante considerar la posición de la muñeca al evaluar la varianza cubital. Esta varianza puede aumentar de 1 a $2 \mathrm{~mm}$ al realizar pronación completa y puño desde una posición neutra de la muñeca. Por lo tanto, al evaluar la varianza cubital deben realizarse radiografías tanto de frente en posición neutra, como en pronación máxima realizando puño (fig. 1). Una varianza cubital negativa o cúbito minus se asocia con la enfermedad de Kienböck y la laxitud ligamentaria del carpo. La varianza cubital positiva o cúbito plus se asocia con impactación cubitocarpiana, lesiones del ligamento lunopiramidal, lesiones del fibrocartílago triangular y artrosis primaria de la ARCD 1 .

El segundo parámetro a evaluar en las radiografías PA de muñeca es el ángulo y la configuración articular radiocubital distal. Basados en el método de Ekenstam², la cavidad sigmoidea del radio posee, como promedio, una inclinación de $7,7^{\circ}$ y la carilla articular del cúbito posee una tangente con inclinación de $22^{\circ 3}$. Sin embargo, esta relación puede encontrarse en ciertas oportunidades paralelas al eje longitudinal del cúbito, o anguladas hacia radial (articulación radiocubital reversa) ${ }^{4}$. Esta última configuración toma especial relevancia durante la osteotomía de acortamiento cubital. En presencia de una ARCD reversa, el acortamiento del cúbito predispone a un pinzamiento (disminución del espacio articular) de la ARCD ${ }^{4}$.

En el caso de sospecharse un pinzamiento dinámico (en situaciones de estrés) de la articulación, este se evidenciará en una radiografía PA con carga. Esta debe hacerse mientras el paciente sostiene una pesa de $2,5 \mathrm{~kg}$ con el hombro en

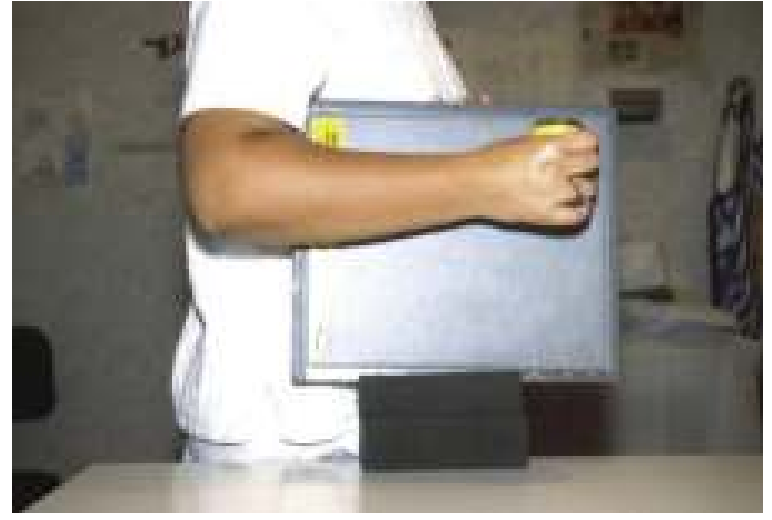

Figura 2 Radiografía en carga para evaluar el pinzamiento (impacto) dinámico radiocubital distal. Posición del paciente, codo contra el tórax con 90 grados de flexión. Carga de $2,5 \mathrm{~kg}$. Incidencia del rayo coronal a la articulación radiocubital distal (ARCD) en posición anatómica. Cortesía del Christine M. Kleinert Institute for Hand \& Microsurgery.

aducción y el codo en $90^{\circ}$ de flexión (fig. 2). De esta manera se hace evidente radiográficamente, en caso de existir, el pinzamiento dinámico radiocubital distal. Esta radiografía es útil también para evaluar el impacto que ocurre entre el cúbito distal y el radio después de procedimientos ablativos de rescate como Darrach o Sauvé-Kapandji (fig. 3).

Finalmente, todo examen radiográfico debe evaluar el estado y desgaste articular, y más teniendo en cuenta el tema que nos compete. La presencia o no de signos radiográficos de artrosis (disminución de la interlínea articular, osteófitos, esclerosis del hueso subcondral...) será fundamental en la toma de decisiones terapéuticas a la hora de abordar el tratamiento de cualquier patología relacionada con la articulación. Las radiografías simples son el pilar de los estudios complementarios a la hora de evaluar la degeneración artrósica de la ARCD.

Otras pruebas de imagen adicionales útiles son la resonancia magnética nuclear, comúnmente utilizada en el diagnóstico de fracturas ocultas, lesiones condrales 


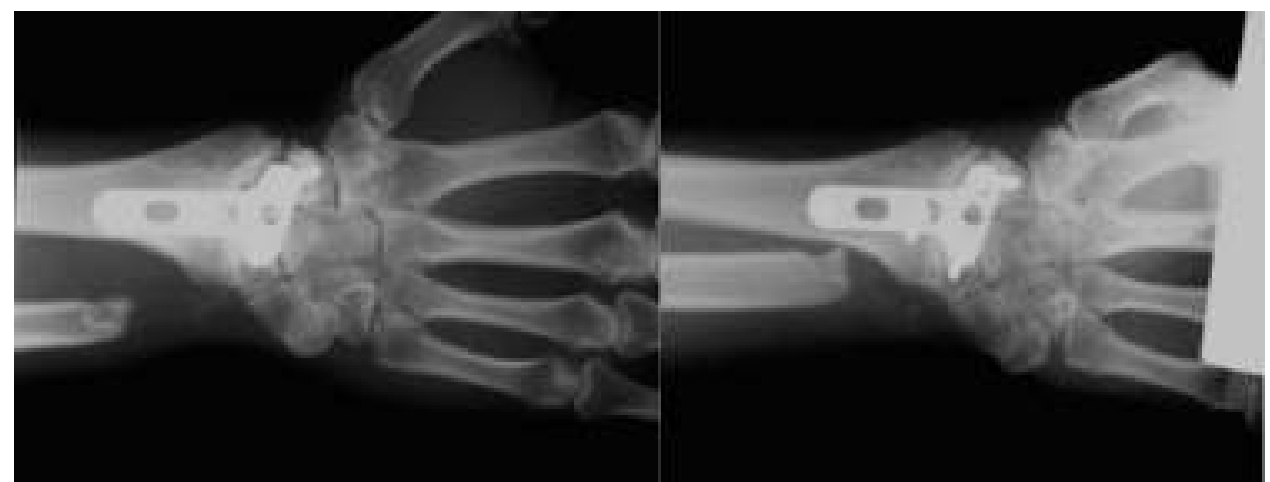

Figura 3 Pinzamiento (impacto) dinámico entre el radio y el cúbito tras la resección distal del cúbito. Cortesía del Christine M. Kleinert Institute for Hand \& Microsurgery.

y lesiones del complejo fibrocartílago triangular y la tomografía axial computarizada (TAC).

La TAC es sobre todo útil en el diagnóstico de fracturas articulares, pero también en el estudio de subluxación y congruencia articular radiocubital distal. La luxación radiocubital distal puede ser evaluada en la radiografía lateral, pero cuando la subluxación es sutil o existe limitación de rotación del antebrazo para lograr un perfil radiográfico estricto, la TAC se considera el estudio de imagen de elección ${ }^{5}$. La TAC permite evaluar la ARCD en el plano coronal sin superposición de estructuras vecinas en los distintos grados de rotación del antebrazo.

A pesar de su utilidad, en el ámbito de la artrosis de la ARCD estas pruebas de imagen adicionales están reservadas más para el estudio prequirúrgico que para el diagnóstico ${ }^{6}$.

\section{Tratamiento de la artrosis de la articulación radiocubital distal}

El tratamiento de la artrosis de la ARCD puede ser conservador o quirúrgico. El objetivo del tratamiento conservador es la disminución del dolor y la optimización de la función articular de manera que la morbilidad de la artrosis pueda ser reducida al mínimo durante el mayor tiempo posible hasta que la cirugía se hace inevitable. El tratamiento conservador incluye medidas de soporte general como inmovilización, analgésicos e inyecciones intraarticulares de esteroides o ácido hialurónico ${ }^{7}$, y debe considerarse siempre antes de indicar un tratamiento más agresivo ${ }^{8}$. En cuanto al tratamiento quirúrgico, se han descrito multitud de procedimientos diferentes que reflejan la variedad del espectro patológico de la $\mathrm{ARCD}^{7}$ :

En los estadios iniciales de la artrosis en los que solo el tercio proximal de la superficie de la articulación está afectado, la osteotomía de acortamiento de cúbito distal puede ser una buena opción de tratamiento con el objetivo de cambiar las superficies de contacto articulares entre la fosa sigmoidea y la carilla articular de la cabeza del cúbito. Usando esta técnica, Scheker y Severo ${ }^{9}$ consiguieron la completa desaparición del dolor en 16 de 32 pacientes tras un seguimiento medio de más de 3 años.

Para el tratamiento de la artrosis en estadios más avanzados, el procedimiento más antiguo descrito es la resección del tercio distal del cubito, técnica popularizada por Darrach en $1912^{10}$. Hasta hace no mucho tiempo, la ARCD era una articulación cuya anatomía y biomecánica no eran bien entendidas, y de ahí que su patología se tratase basándose en criterios con poco fundamento. Al extirpar la porción distal del cúbito, el dolor, efectivamente, puede desaparecer. Sin embargo, aunque estudios antiguos de la técnica mostraron éxito con buenos resultados en más del $80 \%$ de los pacientes analizados ${ }^{11,12}$, otros más recientes con grupos de pacientes más numerosos han mostrado una tasa de fracaso mucho mayor, con serias limitaciones funcionales, especialmente en los sujetos más jóvenes ${ }^{13,14}$. Con este procedimiento, la función de carga del cúbito queda completamente destruida. Además, este proceso en sí mismo es un procedimiento desestabilizador pues, cuando se reseca el cúbito distal, el fibrocartílago triangular, los ligamentos radiocubitales distales y el ECU se separan del cúbito dejando un antebrazo potencialmente inestable que, al final, conducirá a la producción de dolor y chasquidos en el extremo distal remanente del cúbito (fig. 3). Otras complicaciones posibles incluyen la traslación cubital del carpo y la rotura por atrición de tendones extensores ${ }^{11}$.

Otro procedimiento ablativo comúnmente usado es el procedimiento de Sauvé-Kapandji que consiste en la fusión de la cabeza del cúbito al radio distal y la creación de una seudoartrosis del cúbito ${ }^{15}$. Este procedimiento tiene la ventaja de mantener el soporte cubital del carpo y del ECU y preservar el CFCT. Los estudios presentados por Sanders et al. ${ }^{16}$ y Mikkelsen et al. ${ }^{17}$ demostraron una movilidad sin dolor en más de 2 tercios de los pacientes estudiados. Los resultados resultaron ser más satisfactorios en pacientes jóvenes con problemas radiocubitales postraumáticos. Más tarde, Vincent et al. ${ }^{18}$, Rothwell et al. ${ }^{19}$ y Papp et al. ${ }^{20}$ aplicaron la técnica en pacientes con artritis reumatoide y reportaron igualmente resultados satisfactorios. En general, la técnica está indicada en pacientes jóvenes con alta demanda funcional en las muñecas y en pacientes con incompetencia de los ligamentos radiocarpianos, como en la artritis reumatoide ${ }^{21}$. Las complicaciones que se han comunicado incluyen la inestabilidad del extremo distal del cubito remanente, la no unión de la fusión radiocubital y la osificación heterotópica ${ }^{17,18}$.

Debido a la inestabilidad del antebrazo resultante tras estos procedimientos, Bowers en 1985 propuso la artroplastia de hemirresección-interposición ${ }^{22}$, y Watson en 1986 describió la resección «emparejada» de cúbito distal 
(matched distal ulnar resection) ${ }^{23}$, técnicas que preservan el complejo ligamentoso cubitocarpiano. La técnica de Bowers se trata de la resección de la ARCD a nivel de la fosa sigmoidea. Una porción del cúbito se conserva para mantener la integridad del complejo fibrocartílago triangular y una porción de tejido autólogo (pronador cuadrado, flap capsular...) es interpuesto entre el radio y el cúbito para prevenir el impingement (choque) radiocubital. En grupos de pacientes bien seleccionados los resultados de las series publicadas son satisfactorios $22,24,25$. El éxito del procedimiento depende de la integridad del complejo fibrocartílago triangular ${ }^{25}$. Las complicaciones que se han descrito con esta técnica incluyen la impactación estilocarpiana, tendinitis del ECU posquirúrgica, distrofia simpático refleja y neuroma de la rama sensitiva dorsal del nervio cubital ${ }^{22,24}$. En la técnica de Watson, el cúbito distal se reseca formando una larga curva convexa emparejándose con la fosa sigmoidea, sin ninguna interposición entre el radio y el cúbito ${ }^{23}$. El procedimiento elimina la ARCD, pero preserva el complejo fibrocartílago triangular y el mecanismo de soporte cubitocarpiano, elementos estabilizadores. La experiencia con esta técnica está limitada por el seguimiento a largo plazo - por parte del autor principal- de 32 pacientes, de los cuales 24 mostraron buenos o excelentes resultados ${ }^{26}$.

Aunque los resultados de todos estos procedimientos ablativos han resultado ser satisfactorios en muñecas de baja demanda funcional, en el caso de los individuos activos y de alta demanda funcional la norma es el fracaso. El denominador común de estas técnicas es la inestabilidad de la diáfisis del cúbito y su choque contra el radio.

Finalmente, otra alternativa para el tratamiento de la artrosis de la ARCD es el reemplazo protésico del cúbito distal o parte de él (hemiartroplastia), o de toda la ARCD (artroplastia total) mediante prótesis metálicas, de cerámica o de Silastic ${ }^{\circledR}$.

Entre las hemiartroplastias existen técnicas de revestimiento articular del cúbito (Resurfacing First Choice ${ }^{\circledR}$ DRUJ System de Ascension Orthopedics y Eclypse ${ }^{T M}$ de Tornier) y técnicas de recambio protésico total del extremo distal del cúbito (Integra ${ }^{\circledR}$ First Choice ${ }^{\circledR}$ DRUJ y uHead ${ }^{T M}$ de Small Bone Innovations). Eclypse ${ }^{T M}$ es un espaciador de pirocarbono que preserva la estiloides cubital y el CFCT, por lo que conserva los estabilizadores fisiológicos de la ARCD. Su estructura bipolar cubital reduce el desgaste de la articulación mediante el giro del componente articular de pirocarbono alrededor del vástago intramedular insertado en el cúbito. Con la misma filosofía, la prótesis Resurfacing de First Choice ${ }^{\circledR}$ DRUJ System de Ascension Orthopedics también conserva dichos estabilizadores, solo que en su caso se trata de un monobloque en cromocobalto con vástago poroso.

Las prótesis totales de la cabeza de cúbito no solo sustituyen la superficie articular, sino que también reemplazan el resto de estructura de la cabeza cubital. De entre ellas, las previamente comentadas (Integra ${ }^{\circledR}$ First Choice ${ }^{\circledR}$ y uHead ${ }^{T M}$ ) están diseñadas con varios agujeros para el reanclaje de los tejidos blandos y proporcionar supuestamente una mayor estabilidad (fig. 4).

El problema de las técnicas de hemiartroplastia es que tan solo involucran a una de las partes de la ARCD, la carilla articular del cúbito, y con ellas se corre el riesgo de

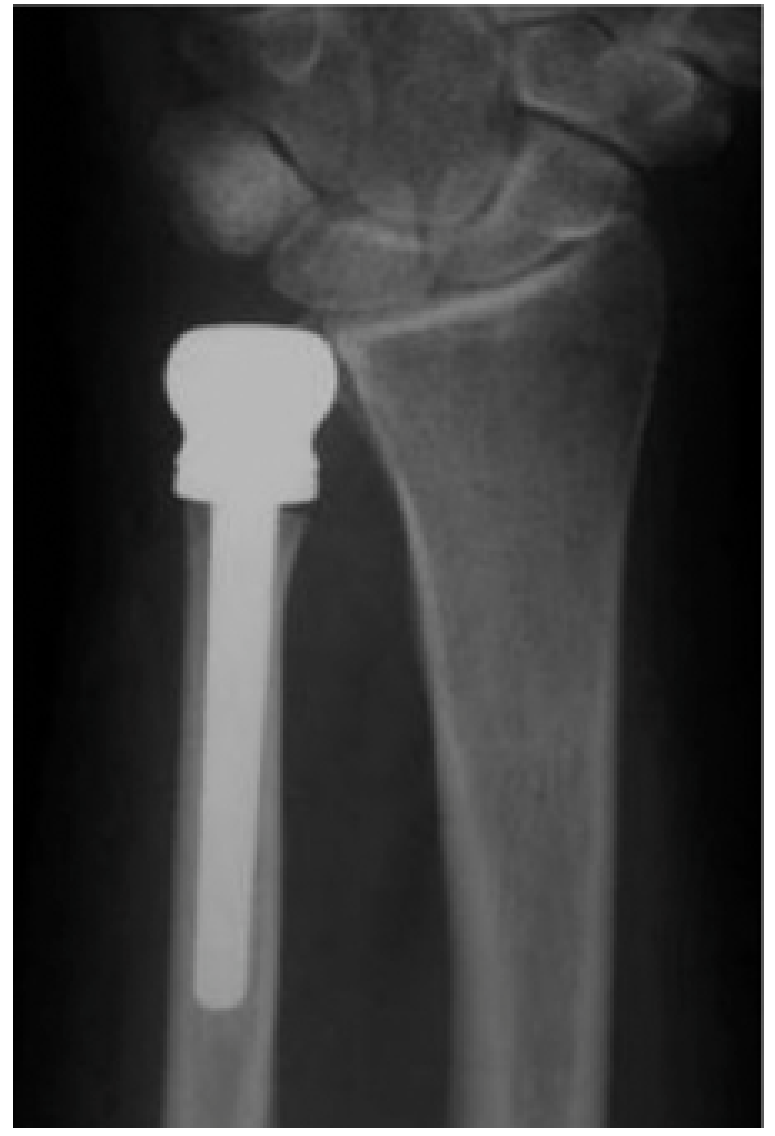

Figura 4 Radiografía de una reconstrucción de la articulación radiocubital distal (ARCD) con una hemiartroplastia. Cortesía del Christine M. Kleinert Institute for Hand \& Microsurgery.

desarrollar cambios degenerativos en la fosa sigmoidea, que se conserva. Aunque a corto plazo los resultados son buenos, no es raro que a la larga los pacientes sufran dolor recurrente e inestabilidad debida al fallo de los tejidos blandos estabilizadores ${ }^{27}$.

En los casos en los que la degeneración artrósica afecte a ambas superficies articulares de la ARCD se debe considerar el recambio total de la articulación. Existen 2 opciones: prótesis no constreñidas y prótesis semiconstreñidas.

En las prótesis no constreñidas, el diseño de la parte articular del implante es el que proporciona la estabilidad a la prótesis remedando la anatomía normal de la articulación. Los tejidos blandos circundantes deben estar intactos o ser reconstruidos concienzudamente para evitar la inestabilidad de la articulación protetizada. Además, suelen necesitar un buen stock óseo para poder ser implantadas. Un ejemplo de estas es la prótesis total de ARCD de Small Bone Innovations (Stability Sigmoid Notch Total DRUJ System ${ }^{T M}$ ) que posee un componente cubital similar a la prótesis parcial uHead ${ }^{T M}$ que se acopla a un implante que reemplaza la fosa sigmoidea formado por una placa metálica con un inserto de polietileno. Una complicación frecuente de estas prótesis es la inestabilidad por distensión de las partes blandas y el desgaste del polietileno ${ }^{7}$.

Las prótesis semiconstreñidas tienen libertad de movimiento en su plano primario, pero movilidad limitada o 


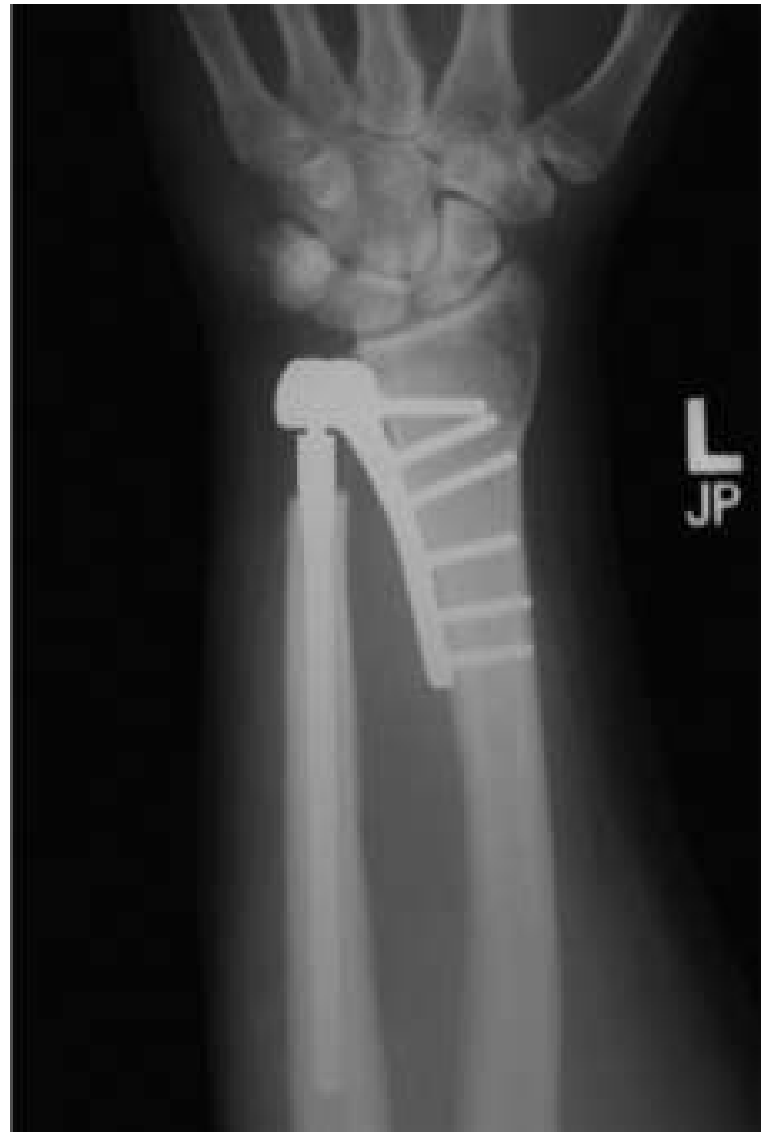

Figura 5 El resultado de una artroplastia total de la articulación radiocubital distal (ARCD) con una prótesis modular semiconstreñida con polietileno de ultraalto peso molecular. Antes del reemplazamiento protésico, el paciente había sido intervenido de una escisión del cúbito distal y presentaba dolor al movilizar la muñeca y el antebrazo desde hacía 2 años. El seguimiento 5 años después de la colocación de la prótesis reveló una pronosupinación completa del antebrazo, así como un completo arco de movilidad tanto de la muñeca como del codo sin dolor. El paciente retomó su previo trabajo 4 meses tras la cirugía. Cortesía del Christine M. Kleinert Institute for Hand \& Microsurgery.

constreñida en el resto de los planos. Son una buena opción de tratamiento cuando, además de artrósica, la ARCD está inestable ${ }^{7}$. Dentro de este concepto se enmarca la prótesis radiocubital distal de Scheker de APTIS, que remplaza la función de la cabeza del cúbito, la fosa sigmoidea del radio y los ligamentos del $\mathrm{CFCT}^{28}$. Esta prótesis permite una estabilidad total, permitiendo al mismo tiempo una pronosupinación completa y adaptándose, además, a los pequeños cambios de longitud axial asociados a la traslación longitudinal fisiológica del radio con respecto al cúbito durante estos movimientos ${ }^{29}$ (fig. 5). El componente cubital de la prótesis de Scheker se trata de un vástago no cementado que se extiende hasta el nivel de la ARCD para ser el centro de rotación de la articulación, en la que se adapta una bola de polietileno que rota libremente sobre el eje axial. Esta bola, a su vez, está contenida en una caja que forma parte de una placa de acero inoxidable que se fija al borde cubital del radio distal. Dentro de esta caja, la bola tiene libre rotación y permite los pequeños cambios de longitud del radio respecto al cúbito durante los movimientos de pronosupinación, pero se mantiene completamente estable en el plano anteroposterior. En el estudio más recientemente publicado, los resultados tras 5 años de seguimiento de esta prótesis en 27 pacientes mostraron una importante mejoría en la fuerza de agarre, en la fuerza de levantamiento de peso, en el rango de movilidad y en el dolor. De ellos, un $30 \%$ presentaron complicaciones como tendinitis del ECU, formación ectópica de hueso y un aflojamiento de uno de los componentes del implante 28 .

Visto todo esto, no es difícil comprender que el mejor conocimiento de la anatomía y la biomecánica de la ARCD, los avances en la tecnología del diseño de los implantes y la presencia de más estudios con seguimiento a largo plazo y mayor número de pacientes, hayan convertido al reemplazo protésico en la tendencia actual para el tratamiento de la artrosis de la ARCD. Probablemente en un futuro muy cercano será el tratamiento estándar y se quedarán atrás los obsoletos procedimientos ablativos, solo justificables en ciertas comunidades sin acceso a las prótesis.

\section{Conflicto de intereses}

El Dr. Scheker es inventor de la prótesis radiocubital distal de Aptis Medical y propietario de Aptis Medical. El resto de los autores declaran no tener ningún conflicto de intereses.

\section{Bibliografía}

1. Katayama T, Ono H, Suzuki D, Akahane M, Omokawa S, Tanaka Y. Distribution of primary osteoarthritis in the ulnar aspect of the wrist and the factors that are correlated with ulnar wrist osteoarthritis: A cross-sectional study. Skeletal Radiol. 2013;42:1253-8.

2. Af Ekenstam F, Haegert CG. Anatomical studies on the geometry and stability of the distal radioulnar joint. Scand J Plast Reconstr Surg. 1985;19:17-25.

3. Sagerman SD, Zogby RG, Palmer AK, Werner FW, Fortino MD. Relative articular inclination of the distal radioulnar joint: A radiographic study. J Hand Surg Am. 1995;20:597-601.

4. Tolat AR, Sanderson PL, de Smet L, Stanley JK. The gymnast's wrist: Acquired positive ulnar variance following chronic epiphyseal injury. J Hand Surg Br. 1992;17:678-81.

5. Mino DE, Palmer AK, Levinsohn EM. Radiography and computerized tomography in the diagnosis of incongruity of the distal radio-ulnar joint. J Bone Joint Surg. 1985;67:247-52.

6. Nacke E, Paksima N. The evaluation and treatment of the arthritic distal radioulnar joint. Bull Hosp Jt Dis. 2015;73:141-7.

7. IFSSH Scientific Committee Report on Degenerative ArthritisDistal Radioulnar Joint. Part 2. Management of Distal Radioulnar Joint Degeneration. Report submitted April 2014.

8. Zimmerman RM, Kim JM, Jupiter JB. Arthritis of the distal radioulnar joint: From Darrach to total joint arthroplasty. J Am Acad Orthop Surg. 2012;20:623-32.

9. Scheker LR, Severo A. Ulnar shortening for the treatment of early post-traumatic osteo-arthritis at the distal radioulnar joint. J Hand Surg Br. 2001;26:41-4.

10. Darrach W. Partial excision of the lower shaft of the ulna for deformity following Colles' fracture. Clin Orthop. 1992;275:3-4.

11. Hartz CR, Beckenbaugh RD. Long-term results of resection of the distal ulna for post-traumatic conditions. J Trauma. 1979;19:219-26. 
12. Dingman PV. Resection of the distal end of the ulna (Darrach operation); an end result study of twenty four cases. J Bone Joint Surg Am. 1952;34:893-900.

13. Bieber EJ, Linscheid RL, Dobyns JH, Beckenbaugh RD. Failed distal ulna resections. J Hand Surg Am. 1988;13:193-200.

14. Bell MJ, Hill RJ, McMurtry RY. Ulnar impingement syndrome. J Bone Joint Surg Br. 1985;67:126-9.

15. Sauve L, Kapandji M. Nouvelle technique de traitment chirurgical des luxations recidivantes isolees de l'extremite inferieure du cubitus. J Chir (Paris). 1936;47:589-94.

16. Sanders RA, Frederick HA, Hontas RB. The Sauve-Kapandji procedure: A salvage operation for the distal radioulnar joint. J Hand Surg Am. 1991;16:1125-9.

17. Mikkelsen SS, Lindblad BE, Larsen ER, Sommer J. SauveKapandji operation for disorders of the distal radioulnar joint after Colles' fracture. Good results in 12 patients followed for 1.5-4 years. Acta Orthop Scand. 1997;68:64-6.

18. Vincent KA, Szabo RM, Agee JM. The Sauve-Kapandji for reconstruction of the rheumatoid distal radioulnar joint. J Hand Surg Am. 1993;18:978-83.

19. Rothwell AG, O’Neill L, Cragg K. Sauve-Kapandji procedure for disorders of the distal radioulnar joint: A simplified technique. J Hand Surg Am. 1996;21:771-7.

20. Papp M, Papp L, Lenkei B, Karolyi Z. Long term results of the Sauvé-Kapandji procedure in the rheumatoid wrist. Act Orth Bel. 2013;79:655-9.
21. Lluch A. The Sauvé-Kapandji procedure. J Wrist Surg. 2013;2: 33-40.

22. Bowers WH. Distal radioulnar joint arthro- plasty: The hemiresection-interposition technique. J Hand Surg Am. 1985;10:169-78.

23. Watson HK, Ryu JY, Burgess RC. Matched distal ulnar resection. J Hand Surg Am. 1986;11:812-7.

24. Bain GI, Pugh DM, MacDermid JC, Roth JH. Matched hemiresection interposition arthroplasty of the distal radioulnar joint. J Hand Surg Am. 1995;20:944-50.

25. Minami A, Kaneda K, Itoga H. Hemiresection-interposition arthroplasty of the distal radioulnar joint associated with repair of the triangular fibrocartilage complex lesions. J Hand Surg Am. 1991;16:1120-5.

26. Watson HK, Gabuzda GM. Matched distal ulna resection for posttraumatic disorders of the distal radioulnar joint. J Hand Surg Am. 1992;7:724-30.

27. Willis AA, Berger RA, Cooney WP 3rd. Arthroplasty of the distal radioulnar joint using a new ulnar head endoprosthesis: Preliminary report. J Hand Surg Am. 2007;32:177-89.

28. Savvidou C, Murphy E, Mailhot E, Jacob S, Scheker LR. Semiconstrained distal radioulnar joint prosthesis. J Wrist Surg. 2013;2:41-8.

29. Degreef I, de Smet L. The Scheker distal radioulnar joint arthroplasty to unravel a virtually unsolvable problem. Acta Orthop Belg. 2013;79:141-5. 